\title{
De la Televisión a la Red. Diferencias entre el Subtitulado Profesional y el Amateur
}

\author{
M. José Rodríguez Campillo* \\ Universitat Rovira i Virgili, Tarragona, Spain
}

\begin{abstract}
This work focuses on the analysis of the linguistic differences between TV and Internet subtitling, specifically YouTube subtitling (which we call "amateur"). Spatio-temporal restrictions that apply to captioning reveal the impossibility of carrying out a literal transcript of what is said, since this transcript would not allow the user to view the image and to extract meaning from content in a such a short period of time. In this paper, we analyze the linguistic consequences of those restrictions and of the differences in the way of subtitling. The main contribution of this paper is to bring to light the need to define new criteria for subtitling in order to improve the understanding of television programs.
\end{abstract}

Keywords: Subtitling, television subtitles, YouTube subtitles.

Resumen. Este trabajo se articula en torno al análisis de las diferencias lingüísticas que hay entre el subtitulado televisivo y el subtitulado en Internet, en concreto el de YouTube (al que llamamos "amateur"). Las restricciones espacio-temporales que se aplican al subtitulado revelan la imposibilidad de llevar a cabo una transcripción literal de lo que se está diciendo, ya que esta no permitiría al usuario el visionado de la imagen y el extraer el significado de su contenido en tan poco tiempo. En

\footnotetext{
* Author's address:

Departament de Filologies Romàniques

Universitat Rovira i Virgili

Av. Catalunya 35, 43002 Tarragona, Spain

E-mail josefa.rodriguez@urv.cat
} 
este trabajo, analizamos las consecuencias lingüísticas que se derivan de estas restricciones y de estas diferencias en la forma de subtitular. La idea principal que se deriva de nuestro análisis es la necesidad de definir nuevos criterios de subtitulación que mejoren la comprensión de los programas televisivos.

Palabras clave: Subtitulación, subtítulos televisivos, subtítulos en YouTube.

\section{Introducción}

La cifra de personas con alguna discapacidad auditiva en España gira alrededor del millón (700 millones en todo el mundo, según Google) y, aunque hay que advertir que el grado de sordera no tiene por qué correlacionarse con la modalidad de comunicación, sí que sabemos que ese casi millón de personas con pérdidas auditivas necesita la eliminación de las barreras de comunicación que les impiden el acceso a la información, por necesidad y por derecho.

Los subtítulos para personas sordas (a partir de ahora, SPS) son una de las mejores herramientas para salvar esas barreras de comunicación, pues crean un contenido adicional que es accesible para ellas, añadiendo la dimensión que necesitan para una perfecta comprensión del mensaje.

Por ello, este trabajo se articula en torno al análisis del SPS y, más en concreto, en torno a las diferencias entre el subtitulado que se ofrece en televisión (profesional) y el que se ofrece en la red, en YouTube (amateur).

El objetivo principal de este trabajo es intentar exponer la realidad del SPS en España, en dos de sus manifestaciones: la televisiva y la de Youtube.

De este objetivo principal se derivan otros, como es el analizar el subtitulado televisivo grabado (profesional y amateur) y, a partir de ahí, analizar las posibles diferencias que hubiera en un mismo programa subtitulado por/con estos distintos medios.

El trabajo que presentamos se ha basado en dos tipos de análisis. En primer lugar, se ha llevado a cabo un análisis crítico de la normativa en materia de SPS para poder obtener unas pautas de trabajo claras. En segundo lugar, se ha realizado un estudio empírico que ha partido de la obtención de un corpus de datos procedente de dos medios (Televisión y YouTube) y el análisis del mismo teniendo en cuenta las pautas obtenidas en la primera parte de este estudio. 
El trabajo se ha realizado grabando y posteriormente visionando y analizando un mismo programa televisivo titulado "El Secreto de Puente Viejo".

El criterio fijado para la selección de la muestra que sirve de base a nuestro análisis del SPS ha sido el hecho de que este programa es un éxito televisivo en la actualidad: lo ven cada tarde casi dos millones de espectadores. Además, se hace de él un subtitulado tradicional no directo, en el cual el subtitulador no necesita "improvisar" e incluso puede seguir el guión del programa.

El método utilizado para la selección de la muestra en ambos medios ha sido el de grabar los mismos días (diez) en ambos medios (Televisión y Youtube).

La elección de dichos días ha sido aleatoria, pero haciéndola coincidir con el quinto aniversario en antena de la serie, cosa que demuestra que es un programa consolidado. Se han analizado varios días de los meses de febrero y marzo de 2016.

\section{Subtítulos}

\subsection{Definición de Subtítulo}

Un posible acercamiento a la definición del subtítulo, tal y como lo vamos a trabajar aquí, sería la que ofrece la UNE 153010 (2012: 2): "Conjunto de texto gráfico que muestran en pantalla los discursos orales, la información contextual y los efectos sonoros que se producen en cualquier obra audiovisual".

Es decir, la subtitulación es el "recuento semántico" (Pereira 2005:162), en la parte inferior de la pantalla (normalmente) y en forma impresa, de lo que se emite oralmente en un programa. A veces, si está bien hecho, no solo emite lo que se dice, sino cómo se dice (tono), qué se oye (música o ruidos, en general) e incluso algún que otro elemento más que aparece en la imagen (cartel, carta,...).

\subsection{Clases de Subtítulos}

Ante la diversidad de productos audiovisuales subtitulados, nos hemos visto en la necesidad de clasificar los distintos subtítulos que podemos encontrar en el mercado, según se escoja un criterio u otro para su definición: 
- Según su presentación formal, distinguimos el subtítulo tradicional (el que se hace antes de su emisión por expertos) y el simultáneo (en directo e incluso, a veces, en semi-directo, pues se puede "pre-grabar" el guión y añadir luego el directo-espontáneo del locutor).

- Según criterios lingüísticos, distinguimos el subtitulado intralingüistico (para personas sordas) y el interlingüistico (el subtitulado por antonomasia de películas traducidas: TAV).

- Según criterios técnicos, distinguimos subtitulados abiertos (aparecen siempre, en todos los programas) y cerrados (se pueden añadir cuando se quieran).

- Según el canal de difusión, podemos tener subtítulos de cine, video, DVD, televisión o YouTube, por ejemplo.

\subsection{Características Generales de los Subtítulos}

Todo subtítulo se caracteriza por una serie de peculiaridades y limitaciones (sobre todo técnicas) que el usuario debe conocer, aunque la práctica profesional puede variar de un país a otro, e incluso de una empresa a otra dentro del mismo país (Díaz-Cintas 2005).

Estas peculiaridades de las que nos habla Díaz-Cintas (2005) están recogidas en la normativa AENOR UNE 153010, publicada en 2012 (en 2003 fue la primera). La normativa fue elaborada gracias a un acuerdo entre los usuarios de subtítulos, la administración y otros agentes (empresas de producción, emisoras de televisión, ...), y su propósito principal es la especificación de los requisitos necesarios para una buena subtitulación de programas, tanto en directo como grabados, "estableciendo unos parámetros mínimos en aras de garantizar la calidad y un grado razonable de homogeneidad en la prestación de este servicio" (UNE 2012: 3).

El grueso de su contenido aborda aspectos como la combinación de colores para la identificación de los personajes o el tamaño de los caracteres. Seguidamente, intentamos resumir las características más relevantes de la misma.

Respecto a los aspectos visuales, la normativa AENOR UNE 153010 indica lo siguiente: 
- Posición del subtítulo: Centrado en la parte inferior de la pantalla, excepto cuando oculten información relevante.

- Posición efectos sonoros: En la parte superior derecha, excepto en subtítulos en directo.

- Número de líneas del texto: Como máximo, dos; excepcionalmente tres en subtítulos en directo.

- Líneas de texto estáticas: Se desaconseja la movilidad de los subtítulos por la necesidad de fijar en exceso la mirada del espectador. En directo, a veces, se puede hacer.

- Lineas de personajes: Se deben asignar líneas de texto en los diálogos que sean distintas a cada personaje.

- Número de caracteres por línea: Máximo 37 caracteres por línea.

- Tamaño mínimo caracteres: Han de ser legibles por personas con visión normal y acoplados (por software) al tamaño de la pantalla.

- Tamaño máximo caracteres: El tamaño que permitan los 37 caracteres por línea.

- Tipografía: Debe responder a criterios de máxima legibilidad.

- Contraste de color entre caracteres y contorno de caja: El contraste ha de ser bueno. Normalmente, color claro sobre fondo oscuro o, al revés, letra oscura sobre fondo claro, pero se prefiere el primero

Por lo que se refiere a los aspectos temporales, las características que destaca la normativa AENOR UNE 153010 son las siguientes:

- Velocidad de exposición: El subtítulo debe seguir el ritmo del hablante y facilitar una lectura cómoda. Siempre que sea necesario, se podrá reducir velocidad, para facilitar la lectura. Estrategia: economizar vocabulario.

- Sincronismo: Las entradas y salidas de los subtítulos deben coincidir, siempre que sea posible, con el movimiento labial, cambios de plano e información sonora. En el subtítulo en directo, es difícil, pero se ha de tratar. 
- Retardo en el subtítulo: Se debe minimizar el retardo entre la emisión del contenido y el subtítulo correspondiente, sobre todo en los directos. El retardo depende de la naturaleza del programa, la tecnología de inserción de subtítulos e incluso del reconocedor de habla, si se utiliza la técnica del rehablado.

En lo referente a la identificación de los personajes, la normativa AENOR UNE 153010 destaca las siguientes características:

- Color: Se debe asignar un color distinto a cada personaje para facilitar así su identificación. El color blanco se debe usar para los personajes "secundarios". Así, el que más diálogo tiene (protagonista) llevará el amarillo y los que menos (secundarios), blanco. El resto de colores se reparten entre los demás personajes. En los documentales, la voz en off en blanco, sobre un fondo negro. El color se ha de intentar mantener siempre.

- Etiquetas: Cuando existe el riesgo de confusión, se deben utilizar etiquetas para la identificación de los distintos personajes. Deben ir antes del subtítulo y deben tener el nombre de la persona o una abreviatura.

- Guiones: Los guiones se utilizarán cuando exista el riesgo de confusión entre personajes que no se puedan diferenciar por colores.

Por lo que respecta a los efectos sonoros, la normativa AENOR UNE 153010 destaca lo siguiente:

- Se suelen subtitular los efectos sonoros necesarios para un buen seguimiento de la trama argumental.

- En directo, se han de hacer siempre que sea posible.

- Se han de sincronizar y adecuarse al ritmo del lenguaje audiovisual, respetando la intención narrativa del relato.

- Han de ir entre paréntesis, la primera letra en mayúscula y el resto en minúsculas.

- A veces, dependiendo del audiovisual, pueden ir en rojo o azul, pero lo normal es en blanco.

- Cuando son redundantes, no hace falta incorporarlos. 
- Hay una serie de sustantivos con efectos sonoros que se suelen utilizar: Disparo, NO Dispara.

- El subtitulado de efectos sonoros se debe referir a su emisión y no a su recepción: "Suena un río" y no "Se oye un río".

Las características que hacen referencia a la información contextual y voz en off, según la normativa AENOR UNE 153010, son las siguientes:

- Se debe subtitular la información contextual para informar sobre las condiciones de las locuciones de los personajes, sobre todo para que el usuario con problemas de audición pueda obtener toda la información pertinente.

- En subtítulos en directo y semi-directo se ha de hacer siempre que se pueda.

- Se deben presentar en mayúsculas y entre paréntesis: (IRÓNICO).

- Deben aparecer delante del texto al que se aplican.

- Deben aparecer en el mismo momento en el que se producen: deben ir sincronizados.

- En caso de voces en off, el subtítulo debe ir en cursiva, si la tecnología utilizada lo permite.

En lo referente a la música y las canciones, la normativa AENOR UNE 153010 indica que se debe hacer lo siguiente:

- La música y las canciones se deben subtitular si son importantes para ayudar al espectador a comprender mejor la trama.

- Se ha de poner el tipo de música: (Música rock).

- Se ha de poner la sensación que transmite: (Música tranquila).

- Se ha de identificar la pieza, si es necesario: (Quinta sinfonía de Beethoven).

- Las letras de las canciones también se deben subtitular si son importantes para que el espectador comprenda la trama.

- Las canciones subtituladas se han de marcar utilizando el símbolo de una nota musical o la almohadilla al principio de cada subtítulo de la canción y en el subtítulo final, uno al inicio y uno al cierre. 
- En las canciones que cantan distintos personajes, debe utilizarse la misma técnica de identificación de los personajes (colores distintos).

Finalmente, en lo referente a los criterios editoriales, la normativa AENOR UNE 153010 indica lo siguiente:

- La división del texto se ha de hacer aprovechando las pausas interpretativas y silencios.

- También se pueden aprovechar las pausas gramaticales o signos de puntuación.

- Las palabras son indivisibles, no se pueden poner en dos líneas distintas.

- Los puntos suspensivos hay que utilizarlos de acuerdo con las normas gramaticales y no para dividir frases en varios subtítulos.

- Se deben seguir los criterios gramaticales y ortográficos de la RAE.

- El habla específica de algún personaje debe mostrarse solo cuando esas palabras mal pronunciadas o esas incorrecciones gramaticales o regionales conlleven información relevante para el seguimiento de la trama. Entonces, se han de poner en forma literal y en cursiva.

- Se han de poner las abreviaturas y símbolos que se puedan abreviar si no, deben escribirse enteros

- Con respecto a la numeración, se deben escribir con letra los ordinales o cardinales del cero al diez, inclusive, y los sustantivos de significación numeral.

- Los subtítulos deberían ser literales, conformes a la locución o a su sentido exacto y propio.

- Los subtítulos (sobre todo los que se hacen en directo) deben alcanzar la máxima precisión en función de las posibilidades tecnológicas del momento. 


\section{Subtitulado en Televisión y Youtube: Análisis Contrastivo}

En este trabajo analizamos el subtitulado tradicional, también llamado grabado. Este subtitulado es específico para personas sordas y se suele ofrecer en cerrado. En concreto, nos centramos en el subtitulado que nos ofrecen dos canales distintos de difusión: la Televisión y YouTube.

El tipo de programas analizado permite un subtitulado grabado, preparado con antelación. Este subtitulado no se realiza en el mismo momento de su emisión.

A la hora de subtitular este tipo de programas, la televisión y YouTube optan por vías parecidas aunque con algunas particularidades. La televisión no permite un subtitulado realizado por el público general, mientras YouTube sí que lo permite. Por eso, hemos llamado al subtitulado televisivo profesional $\mathrm{y}$ al de YouTube amateur.

Como refleja la Tabla 1, hemos analizado más de 9 horas de programa con subtítulos grabados.

\begin{tabular}{|c|c|c|c|}
\hline CAPÍTULO & DIA & MINUTOS & $\%$ \\
\hline $\mathbf{1 2 6 7}$ & $19-02-2016$ & 54,31 & $10 \%$ \\
\hline $\mathbf{1 2 6 8}$ & $22-02-2016$ & 54,21 & $10 \%$ \\
\hline $\mathbf{1 2 6 9}$ & $23-02-2016$ & 54,27 & $10 \%$ \\
\hline $\mathbf{1 2 7 0}$ & $\mathbf{2 4 - 0 2 - 2 0 1 6}$ & $\mathbf{5 4 , 4 1}$ & $\mathbf{1 0} \%$ \\
\hline $\mathbf{1 2 7 1}$ & $25-02-2016$ & 53,56 & $9,9 \%$ \\
\hline $\mathbf{1 2 7 2}$ & $26-02-2016$ & 53,52 & $9,9 \%$ \\
\hline $\mathbf{1 2 7 3}$ & $29-02-2016$ & 55,05 & $10,10 \%$ \\
\hline $\mathbf{1 2 7 4}$ & $02-03-2016$ & 54,36 & $10 \%$ \\
\hline $\mathbf{1 2 7 5}$ & $03-03-2016$ & 55,37 & $10,20 \%$ \\
\hline $\mathbf{1 2 7 6}$ & $04-03-2016$ & 53,50 & $9,9 \%$ \\
\hline & & $\begin{array}{c}\mathbf{5 4 2 , 5 6} \\
(\mathbf{9} \text { horas })\end{array}$ & $\mathbf{1 0 0} \%$ \\
\cline { 3 - 4 } & & &
\end{tabular}

Tabla 1. Capítulos, días y horas de subtitulado en "El secreto de Puente Viejo". 
En la Tabla 2 puede observarse que de esas 9 horas de análisis, hemos recogido 6.857 palabras pronunciadas/emitidas en el capítulo 1270 -que es el que tomamos de base para los ejemplos (las que dicen los actores en el programa, el $100 \%$ de nuestra muestra). De esas 6.857 palabras, en el subtitulado en televisión se recogen 6.597 (el 96\% del total), mientras que en YouTube se transcriben 6.813 (el 99\%). Por tanto, en el paso del audio al subtitulo solo perdemos 260 palabras en los subtítulos televisivos y 44 en los de YouTube: todo un lujo, si lo comparamos con la subtitulación en directo, donde las personas sordas pueden llegar a perderse más del $52 \%$ de la información (según datos de un muestreo/análisis nuestro hecho con anterioridad sobre subtitulación en directo).

\begin{tabular}{|c|c|c|}
\hline FORMATO & PALABRAS & $\%$ \\
\hline Oral & 6857 & $100 \%$ \\
\hline Subtitulado por TV & 6597 & $96,20 \%$ \\
\hline Subtitulado por YouTube & 6813 & $99,36 \%$ \\
\hline
\end{tabular}

Tabla 2. Capítulo 1270 .

Estos datos reflejados en la Tabla 2 son de alguna forma engañosos. Esa subtitulación en YouTube tan atractiva no es tan perfecta como los números parecen indicar pues, muchas de las palabras contabilizadas no tienen significado alguno en castellano. Como muestra la Tabla 3 , si ajustamos los números, eliminando las palabras sin sentido, los resultados muestran que YouTube subtitula aproximadamente el $79 \%$ de lo dicho por los actores.

\begin{tabular}{|c|c|c|}
\hline TOTAL PALABRAS & $\begin{array}{c}\text { PALA BRAS } \\
\text { ENTENDIDAS }\end{array}$ & $\begin{array}{c}\text { PEQUENOS } \\
\text { SINTAGMAS }\end{array}$ \\
\hline \multirow{3}{*}{$6 \mathbf{6 8 1 3}$} & 5834 & 5414 \\
\cline { 2 - 3 } & 1023 menos & $\mathbf{1 4 4 3}$ menos \\
\cline { 2 - 3 } & $85 \%$ & $79 \%$ \\
\hline
\end{tabular}

Tabla 3. Youtube. 
La Tabla 4 muestra un ejemplo de esa transcripción de YouTube en la que, las palabras, a veces, no se entienden.

\begin{tabular}{|c|c|c|}
\hline ORAL & TV & YOUTUBE \\
\hline Que no me tutee & Que no me tutee & Que no me to t \\
\hline Perdone, & Perdone, & arzón \\
\hline la sigo viendo & la sigo viendo & la sigo siendo \\
\hline $\begin{array}{c}\text { Portando ligas y satenes } \\
\text { rojos }\end{array}$ & $\begin{array}{c}\text { Portando ligas y satenes } \\
\text { rojos }\end{array}$ & $\begin{array}{c}\text { portando // elija sisa } \\
\text { tenerlo }\end{array}$ \\
\hline $\begin{array}{c}\text { Escuche, niña } \\
\text { Eso no nos importa }\end{array}$ & $\begin{array}{c}\text { Escuche, niña no nos importa } \\
\text { escuchÉ, niña }\end{array}$ & $\begin{array}{c}\text { eso es lo más } \\
\text { importante }\end{array}$ \\
\hline $\begin{array}{c}\text { algo que usted ha } \\
\text { perdido }\end{array}$ & $\begin{array}{c}\text { algo que usted ha perdido } \\
\text { dado que usted ha } \\
\text { perdido }\end{array}$ \\
\hline $\begin{array}{c}\text { para comprar el cariño } \\
\text { de nadie }\end{array}$ & $\begin{array}{c}\text { para comprar el cariño de } \\
\text { nadie }\end{array}$ & $\begin{array}{c}\text { para dunga el } / / \\
\text { cariño de nadie }\end{array}$ \\
\hline
\end{tabular}

Tabla 4. Un ejemplo.

A continuación, presentamos los distintos aspectos que, según la normativa AENOR UNE 153010, debe cumplir todo buen subtitulado. Enumeraremos las distintas características agrupadas en bloques diferentes y a partir de ellas, especificaremos si el subtítulo televisivo y el de YouTube cumplen o no el requisito especificado.

En primer lugar, la Tabla 5 recoge los datos del cumplimiento de la normativa AENOR UNE 153010 en lo referente a los aspectos visuales de los subtítulos. 


\begin{tabular}{|l|c|c|}
\hline & TV & YOUTUBE \\
\hline Centrado en la parte inferior de la pantalla & SÍ & SÍ \\
\hline Efectos sonoros en la parte superior derecha & SÍ & NO \\
\hline Como máximo, dos líneas & SÍ & SÍ \\
\hline Se desaconseja la movilidad de los subtítulos & SÍ & SÍ \\
\hline Se deben asignar líneas distintas a cada personaje & SÍ & NO \\
\hline Máximo 37 caracteres & SÍ & SÍ \\
\hline Han de ser legibles por personas con visión normal & SÍ & SÍ \\
\hline El tamaño que permitan los 37 caracteres por línea & SÍ & SÍ \\
\hline Debe responder a criterios de máxima legibilidad & SÍ & SÍ \\
\hline El contraste ha de ser bueno & SÍ & SÍ \\
\hline
\end{tabular}

Tabla 5. Aspectos visuales.

La Tabla 6 presenta el grado de cumplimiento de la normativa AENOR UNE 153010 en lo referente a los aspectos temporales de la subtitulación en televisión y YouTube.

\begin{tabular}{|l|c|c|}
\hline & TV & YOUTUBE \\
\hline $\begin{array}{l}\text { El subtítulo debe seguir el ritmo del hablante y facilitar } \\
\text { una lectura cómoda }\end{array}$ & SÍ & NO \\
\hline El subtítulo debe estar sincronizado & SÍ & NO \\
\hline $\begin{array}{l}\text { Se debe minimizar el retardo entre la emisión del } \\
\text { contenido y el subtítulo correspondiente }\end{array}$ & SÍ & NO \\
\hline
\end{tabular}

Tabla 6. Aspectos temporales.

La Tabla 7 presenta los resultados referentes al cumplimiento de la normativa AENOR UNE 153010 en lo referente a la identificación de personajes en la subtitulación profesional y amateur. 


\begin{tabular}{|l|c|c|}
\hline Se debe asignar un color distinto a cada personaje & SÍ & NO \\
\hline El color se ha de intentar mantener siempre. & SÍ & NO \\
\hline $\begin{array}{l}\text { El que más diálogo tiene (protagonista) llevará el } \\
\text { amarillo y los que menos (secundarios), blanco. El } \\
\text { resto de colores se reparten entre los demás }\end{array}$ & SÍ & NO \\
\hline $\begin{array}{l}\text { Cuando existe el riesgo de confusión, utilizar } \\
\text { etiquetas para la identificación de los distintos } \\
\text { personajes. }\end{array}$ & SÍ & NO \\
\hline $\begin{array}{l}\text { Deben ir antes del subtítulo y deben tener el nombre } \\
\text { de la persona o una abreviatura }\end{array}$ & NO & NO \\
\hline $\begin{array}{l}\text { Los guiones se utilizarán cuando exista el riesgo de } \\
\text { confusión entre personajes que no se puedan } \\
\text { diferenciar por colores }\end{array}$ & A & NO \\
\hline
\end{tabular}

Tabla 7. Identificación de personajes.

La Tabla 8 analiza el contraste entre televisión y Youtube en lo referente al cumplimiento de la normativa AENOR UNE 153010 en lo que respecta a los aspectos sonoros del subtitulado.

\begin{tabular}{|l|c|c|}
\hline & TV & YOUTUBE \\
\hline $\begin{array}{l}\text { Subtitular los efectos sonoros necesarios para un } \\
\text { buen seguimiento de la trama argumental }\end{array}$ & SÍ & NO \\
\hline $\begin{array}{l}\text { Se han de sincronizar y adecuarse al ritmo del } \\
\text { lenguaje }\end{array}$ & SÍ & NO \\
\hline $\begin{array}{l}\text { Han de ir entre paréntesis, la primera letra en } \\
\text { mayúscula }\end{array}$ & SÍ & NO \\
\hline $\begin{array}{l}\text { A veces, pueden ir en rojo o azul, pero normal en } \\
\text { blanco }\end{array}$ & NO & NO \\
\hline $\begin{array}{l}\text { Cuando son redundantes, no hace falta } \\
\text { incorporarlos }\end{array}$ & NO & NO \\
\hline Hay una serie de sustantivos con efectos sonoros & SÍ & NO \\
\hline $\begin{array}{l}\text { El subtitulado de efectos sonoros se debe referir a } \\
\text { su emisión y NO a la recepción del mismo }\end{array}$ & SÍ & NO \\
\hline
\end{tabular}

Tabla 8. Efectos sonoros. 
La Tabla 9 presenta los resultados referidos a la información contextual en los subtítulos. Podemos observar el grado de cumplimiento de la normativa AENOR UNE 153010 en este aspecto tanto en el subtitulado televisivo como en el subtitulado de YouTube.

\begin{tabular}{|l|c|c|}
\hline & TV & YOUTUBE \\
\hline $\begin{array}{l}\text { Se debe subtitular la información contextual para } \\
\text { informar sobre las condiciones de las locuciones } \\
\text { de los personajes }\end{array}$ & SÍ & NO \\
\hline $\begin{array}{l}\text { Se deben presentar en mayúsculas y entre } \\
\text { paréntesis }\end{array}$ & SÍ & NO \\
\hline Deben aparecer delante del texto al que se aplican & SÍ & NO \\
\hline Deben ir sincronizados & $\begin{array}{c}\text { CASI } \\
\text { SIEMPRE }\end{array}$ & NO \\
\hline
\end{tabular}

Tabla 9. Información contextual.

La Tabla 10 establece el contraste entre los dos tipos de subtitulación en lo referente a las música y las canciones y refleja hasta qué punto la televisión y YouTube cumplen con la normativa AENOR UNE 153010.

\begin{tabular}{|l|c|c|}
\hline & TV & YOUTUBE \\
\hline $\begin{array}{l}\text { Música y canciones se deben subtitular si son } \\
\text { importantes }\end{array}$ & A VECES & NO \\
\hline Poner el tipo de música: (Música rock) & NO & NO \\
\hline $\begin{array}{l}\text { Poner la sensación que transmite: (Música } \\
\text { tranquila) }\end{array}$ & NO & NO \\
\hline Identificar la pieza: (Quinta sinfonía de Beethoven) & NO & NO \\
\hline $\begin{array}{l}\text { Las letras de las canciones se deben subtitular si son } \\
\text { importantes para que el espectador comprenda la } \\
\text { trama }\end{array}$ & AECES & NO \\
\hline $\begin{array}{l}\text { Las canciones subtituladas se han de marcar } \\
\text { utilizando el símbolo de la almohadilla, al principio } \\
\text { de cada subtítulo y en el subtítulo final, uno al } \\
\text { inicio y uno al cierre }\end{array}$ & Sí & NO \\
\hline $\begin{array}{l}\text { En canciones que cantan distintos personajes, debe } \\
\text { utilizarse misma técnica de identificación de } \\
\text { personajes (colores distintos) }\end{array}$ & SÍ & NO \\
\hline
\end{tabular}

Tabla 10. Música y Canciones. 
Finalmente, la Tabla 11 presenta los resultados referentes al seguimiento de la normativa AENOR UNE 153010 en lo referente a sobre los criterios editoriales de la subtitulación.

\begin{tabular}{|l|c|c|}
\hline & TV & YOUTUBE \\
\hline $\begin{array}{l}\text { La división del texto se ha de hacer aprovechando } \\
\text { las pausas interpretativas y silencios }\end{array}$ & SÍ & NO \\
\hline $\begin{array}{l}\text { También se pueden aprovechar las pausas } \\
\text { gramaticales o signos de puntuación }\end{array}$ & SÍ & SÍ \\
\hline $\begin{array}{l}\text { Las palabras son indivisibles, no poner en líneas } \\
\text { distintas }\end{array}$ & SÍ & NO \\
\hline $\begin{array}{l}\text { Los puntos suspensivos hay que utilizarlos de } \\
\text { acuerdo con las normas gramaticales y no para } \\
\text { dividir frases en varios subtítulos }\end{array}$ & SÍ & NO \\
\hline $\begin{array}{l}\text { Seguir criterios gramaticales y ortográficos de RAE } \\
\text { El habla específica de algún personaje debe } \\
\text { mostrarse solo cuando conlleven información } \\
\text { relevante }\end{array}$ & A VECES & NO \\
\hline $\begin{array}{l}\text { Se ha de poner en forma literal y en cursiva } \\
\text { Se han de poner abreviaturas y símbolos si se puede }\end{array}$ & A VECES & NO \\
\hline $\begin{array}{l}\text { Numeración: escribir con letra los } \\
\text { ordinales/cardinales del cero al diez, inclusive, y los } \\
\text { sustantivos de significación numeral }\end{array}$ & SÍ & A VECES \\
\hline $\begin{array}{l}\text { Subtítulos deberían ser literales, conformes a la } \\
\text { locución }\end{array}$ & A VECES & NO \\
\hline
\end{tabular}

Tabla 11. Criterios Editoriales.

Somos conscientes de que las restricciones espacio-temporales que se aplican a cualquier subtitulado revelan la imposibilidad de llevar a cabo una transcripción literal de lo que se está diciendo, ya que esta no permitiría al usuario el visionado de la imagen y el extraer el significado de su contenido en tan poco tiempo. Sin embargo, y con la normativa de AENOR, disponemos de unas buenas directrices para que el subtitulado sea de ayuda a las personas con problemas de audición.

Quedan algunas cosas por mejorar, sobre todo en YouTube, como la cronometración (la Universidad Carlos III de Madrid está trabajando en ello), 
los signos de interrogación y exclamación (de los que solo se pone el final en televisión y en YouTube no), algunos acentos que, a veces, se ponen en exceso y llegan a confundir al espectador, etc.

\section{Conclusiones}

El objetivo de este trabajo era el de exponer la realidad del SPS en España. También queríamos analizar el subtitulado en Televisión y en Youtube para ver las posibles diferencias entre ellos, sobre todo para intentar ofrecer soluciones más o menos sistematizadas para la mejora de la subtitulación española, independientemente del medio que se utilice para ello.

Los resultados de nuestro análisis indican que, a pesar de que la mayoría de los programas disponen de un servicio de subtitulado para sordos, la calidad del mismo no garantiza, en todos los casos, la inteligibilidad de los contenidos para estas personas con discapacidad auditiva.

Así, a partir de nuestro análisis, concluimos que hay que revisar los criterios del subtitulado que se emplean en la actualidad y que necesitamos definir nuevos criterios de subtitulación que mejoren la comprensión de los programas televisivos, sobre todo en YouTube.

\section{Bibliografía}

1. Arnáiz Urquiza, V. (2012). Los parámetros que identifican el subtitulado para sordos. Análisis y clasificación. MonTI. Monografías de Traducción e Interpretación, 4: 103-132

2. Díaz Cintas, J. (2005). Teoría y práctica de la subtitulación. Barcelona: Ariel.

3. Gottlieb, H. (1997). Subtitles, Translation \& Idioms, Tesis doctoral, University of Copenhague, Copenhague.

4. Ivarsson, J. (1992). Subtitling for the Media: A Handbook of an Art. Estocolmo: TransEdit.

5. Ivarsson, J. \& Carroll, M. (1998). Subtitling. Simrishamn: TransEdit.

6. Izard, N. (2001). La subtitulación para sordos del teletexto de Televisión Española. In Lorenzo, L. \& Pereira, A. (eds.), Traducción Subordinada (II), El Subtitulado (inglés-español/galego) (pp. 169-194). Vigo: Servicio de publicacións da Universidade de Vigo. 
7. Karamitroglou, F. (1998). A proposed Set of Subtitling Standard in Europe. Translation Journal, 2(2).

8. Pereira Rodríguez, A.M. (2005). El subtitulado para sordos: estado de la cuestión en España. Quaderns. Revista de traducció, 12: 161-172.

9. VV.AA (1998). Resolución del Parlamento Europeo, de 17 de junio de 1988, sobre lenguajes gestuales para sordos. Diario Oficial, C187: 0236.

10. VV.AA (2012). Subtitulado para personas sordas y personas con discapacidad auditiva. AENOR UNE 153010. Madrid: AENOR.

\section{Author's Biodata}

M. José Rodriguez Campillo es doctora en literatura española por la Universitat Rovira i Virgili. Actualmente es profesora en el Departament de Filologies Romániques de la URV. Es especialista en literatura española del siglo XVII. Su tesis doctoral sobre las Implicaturas en el teatro femenino de los Siglos de Oro constituye un claro ejemplo de la investigación desarrollada por la Dra. Campillo en la que se concede una importancia fundamental a la interdisciplinariedad. La utilización de métodos lingüísticos para el análisis literario es una constante en sus artículos publicados en revistas y volúmenes monográficos. Ha participado en numerosos congresos internacionales y ha organizado reuniones científicas en las que se pretende fomentar la visibilidad de la literatura escrita por mujeres. 